\title{
L'art du métal au Laos
}

Christine Hawixbrock

\section{Citer ce document / Cite this document :}

Hawixbrock Christine. L'art du métal au Laos. In: Bulletin de l'Ecole française d'Extrême-Orient. Tome 87 N¹, 2000. pp. 109-124;

doi : https://doi.org/10.3406/befeo.2000.3472

https://www.persee.fr/doc/befeo_0336-1519_2000_num_87_1_3472

Fichier pdf généré le 08/11/2019 


\section{Résumé}

Christine Hawixbrock

L'art du métal au Laos

Dans cet article, notre propos a été d'étudier une collection de statuettes du Buddha mise au jour lors d'une fouille de sauvetage sur le site de Huay Sa Hua, dans le Sud Laos, près de la ville de Champassak. Ces statues proviennent d'une cache construite ayant servi de dépôt sacré à l'intérieur d'un monastère dont l'ensemble a été fouillé. Cette collection, qui était en partie en cours de pillage avant que la fouille de sauvetage n'ait lieu, comprenait plus de 200 statuettes bouddhiques, dont la majeure partie en bronze, en or et

en argent. Nous nous sommes attachée ici à étudier 108 statuettes en keson dokmai (résine végétale) recouvertes d'une mince feuille d'or ou d'argent. Ces pièces d'orfèvrerie n'ayant pratiquement jamais fait l'objet d'une étude spécifique, il était nécessaire d'en faire l'analyse stylistique et d'en étudier les modes de confection. Certains de ces Buddha portent sur leur socle une courte inscription témoignant de la raison du don de la statuette au monastère et l'un d'eux, daté, permet d'attribuer ces pièces à la fin du XVIle siècle. Ces images de petites dimensions, qui ont probablement dû voyager facilement à travers le pays, pourraient appartenir à l'art de Luang Prabang (Nord Laos). Leurs caractéristiques stylistiques, sensiblement différentes de celles des grands Buddha en bronze de l'époque, permettent d'affiner la datation de l'art bouddhique du Laos.

\section{Abstract \\ Christine Hawixbrock \\ The art of metalwork in Laos}

In this article, our purpose is to study a collection of small Buddhist statues found during a rescue excavation on the site of Huay Sa Hua (south of Laos), located near the city of Champassak. This collection comes from a sacred deposit found inside a monastery which has been completely excavated. The collection, which was partially plundered before the excavation, included more than 200 statues. Most of them are made of bronze, silver and gold. 108 statues made of keson dokmai (vegetate resin) and covered by a thin layer of silver or gold leaf have been studied. Statues such as these have never before been studied. It was therefore necessary to do research on both their style and the process of their manufacture. Some of these Buddhas have small inscriptions on their base, describing the purpose of the gift to the monastery. One of them carries a date which allowed us to date the whole collection to the end of the seventeenth century. This kind of small, easily portable image appears to belong to the art of Luang Prabang. Their stylistic characteristics, slightly different from those of the large Buddhas of the same period, could prove useful for refining the dating of the Buddhist art of Laos. 


\title{
L'art du métal au Laos
}

\author{
Christine HAWIXBROCK
}

\section{Introduction}

Les statuettes que nous allons présenter proviennent de la fouille de sauvetage d'un monastère laotien situé en haut de la falaise qui constitue la rive droite du Mékong, à une centaine de mètres au nord du confluent de la rivière Huay Sa Hua. Ce site a été fouillé par le Programme de recherche en archéologie lao (PRAL) ${ }^{1}$ en 1992 ; il se trouve dans l'enceinte de la ville khmère du VI ${ }^{\mathrm{e}}$ siècle associée au sanctuaire de Vat Phu (sud du Laos). Comme il ne répondait à aucun nom précis, il a été dénommé Huay Sa Hua 1 (HSH-1) pour le différencier des autres chantiers archéologiques que l'équipe menait en parallèle à ce moment-là.

C'est une crue du Mékong, durant la saison des pluies de 1992, qui, en emportant une partie de la berge du fleuve, a révélé l'existence d'une cache, couverte d'une dalle de grès en réemploi, qui provient manifestement d'un site khmer préangkorien démembré, tandis que les parois verticales étaient en brique. Lors de sa découverte, effectuée par l'équipe lors de prospections en bordure du Mékong, cette cache était déjà en cours de pillage. Elle était en effet située sur un terrain privé et avait commencé à être vidée. Les autorités culturelles de la province nous ont alors demandé d'effectuer la fouille de ce site, qui correspond à un monastère bouddhique désaffecté depuis plus d'un siècle. Une publication exhaustive étant prévue prochainement sur l'ensemble des résultats qu'ont donnés ces fouilles, nous ne nous étendrons pas ici sur le contexte archéologique de ces trouvailles. Une première présentation des travaux a été faite à Rome, en 1992, lors de la quatrième conférence de l'Association européenne des archéologues de l'Asie du Sud-Est, et nous renvoyons à la publication correspondante pour les premières données résultant de ces fouilles ${ }^{2}$. Notons simplement que ces statuettes proviennent d'un dépôt de fondation

1. Créé en 1991, le PRAL est co-dirigé par Marielle Santoni (CNRS) et Viengkèo Souksavatdy, directeur de la Division de l'archéologie et des musées, ministère de l'Information et de la Culture de la République démocratique populaire lao. - Le présent travail n'aurait pas été possible sans la collaboration des membres de ce programme ; nous tenons à remercier plus particulièrement Jean-Pierre Message, qui a fait les dessins, classé les décors et restauré chaque Buddha, Viengkèo Souksavatdy, qui a lu et traduit les inscriptions, et Marielle Santoni, grâce à qui cette étude a vu le jour et qui a mis à notre disposition les photographies qui illustrent cet article.

2. Marielle Santoni, Viengkèo Souksavatdy, Denis Defente, Christine Hawixbrock, Jean-Claude Liger, «Excavations at Champassak and Vat Phu (Southern Laos) », dans Roberto Ciarla and Fiorella Rispoli (éd.), Southeast Asian Archaeology 1992, Proceedings of the Fourth International Conference of the South-East Asian Archaeologists, Rome, 28th September - 4th October 1992, Roma, Is.I.A.O., 1997, p. 233-263; voir aussi Viengkèo Souksavatdy, L'archéologie des débuts de l'histoire khmère dans la 
installé probablement sous le sanctuaire principal, et destiné à le sanctifier. La partie est du monastère a été emportée par les crues successives du Mékong, mais la partie ouest, conservée, a été fouillée entièrement ${ }^{3}$. Ce dépôt de fondation n'était accessible que depuis la falaise en bordure du Mékong, par une cavité pratiquée par les pilleurs dans la paroi en brique est.

Pour plus de clarté, nous appellerons ces statuettes, toutes d'obédience bouddhique, des keson dokmai («pollen de fleurs », syntagme désignant la résine végétale qui compose leur âme). Elles ne constituent cependant pas l'ensemble du trésor mis au jour à l'intérieur de cette cache. Une centaine de plaques de petites dimensions, en or ou en argent - qu'on appelle également des Saintes Empreintes (Phra Patima) -, en faisaient partie ainsi que quelques Buddha en bronze de plus grande taille, des Buddha en résine et des Phra Kachayana (disciple du Buddha et saint bouddhiste particulièrement vénéré en Asie du Sud-Est) en résine, argent, plomb, et bronze.

Ces statuettes, ou du moins celles retrouvées en place, avaient dans la majorité des cas le visage tourné vers l'est, ce qui est normal dans un contexte bouddhique. Les plaques étaient appuyées contre les parois de la cache.

Nous avons choisi dans cet article de présenter uniquement les statuettes en keson dokmai dans la mesure où elles constituent un ensemble cohérent, datant de la même période. Les Saintes Empreintes sont en effet probablement plus anciennes et réutilisent la stylistique khmère bouddhique. En tout, cent huit exemplaires de ces statuettes ont été retrouvés, à l'état complet ou fragmentaire. Mais nous savons qu'à l'origine elles étaient plus nombreuses, le pillage étant déjà en cours lorsque les fouilles ont débuté.

À notre connaissance, aucune collection de ce type n'a encore été étudiée par les historiens de l'art, d'où le peu de références sur lesquelles nous avons pu nous appuyer et la nécessité de présenter ces pièces en détail.

\section{État de conservation actuel}

De part leur constitution, ces statuettes sont très fragiles. Leur âme est constituée d'une pâte faite à base de résine et de fleurs pilées, elle est donc assez friable. Ce mélange est coulé à l'intérieur d'une feuille d'or ou d'argent, parfois mixte (Buddha en or, socle en argent), de faible épaisseur. De ce fait, et malgré leur conservation dans un contexte clos, relativement protégé bien qu'il ait eu à subir les crues répétées du Mékong, ces statuettes ont assez souffert. Peu d'entre elles nous sont parvenues intactes ou complètes. La majorité des fragments conservés est surtout constituée des socles, le corps des Buddha ayant dans de nombreux cas disparu. Si les revêtements en or ont la plupart du temps résisté, ceux en argent, beaucoup plus fragiles, n'existent plus, le plus souvent, qu'à l'état de traces. Cependant, le type de technique utilisée pour leur fabrication (sur laquelle nous reviendrons plus loin avec plus de précision) et le nombre des individus préservés est suffisamment élevé pour nous permettre une étude précise de ces pièces.

\section{Présentation des principales caractéristiques iconographiques}

Étant donné le nombre assez conséquent des pièces que nous présentons ici, il ne nous est pas possible de donner une description précise de chacune d'entre elles. Une base de donnée iconographique systématique a été établie par nos soins, qui sera publiée dans la

région de Champassak, (mémoire de DEA présenté à l'EPHE, IVc section, sous la direction de Michel Terrasse), Paris, 1997.

3. Marielle Santoni et al., art. cité, fig. 20 à 22 . 
monographie d'ensemble. Chaque Buddha a ainsi reçu un numéro qui permet de 1'identifier (exemple: HSH1-92.001). Nous avons donc choisi de donner ici une description générale des pièces, avec les constantes iconographiques que l'on peut constater, ainsi que les divergences.

Ces pièces sont toutes de petite taille : les plus grandes font environ $17 \mathrm{~cm}$ de haut, celles de taille moyenne avoisinent les $13 \mathrm{~cm}$ et sont les plus nombreuses, tandis que les plus petites, qui font autour de 8 à $10 \mathrm{~cm}$ de haut, en fonction de la taille de leur flamme ou bulbe, qui peut être parfois très imposante, sont les moins nombreuses.

Toutes les statuettes sont figurées en position assise, jambes repliées l'une sur l'autre. Les mudrā effectuées sont invariablement les mêmes : Prise de la Terre à témoin pour la main droite (bhümiśparsamudrā, également appeléc la Victoire sur Māra, ou māravijaya) et Méditation (samādhi) pour la main gauche. L'épaule droite est toujours découverte, la sanghātī (écharpe) est pliée sur l'épaule gauche et retombe jusqu'au bas du dos, avec, dans la plupart des cas, l'indication des plis en bordure inférieure antérieure et postérieure par un liseré simple ou double horizontal, ondulé ou en pointe. Une seule statuette diffère, par l'inversion de l'uttarāsaniga (manteau monastique) qui lui laisse l'épaule gauche découverte tandis que la sanghātī est pliée sur l'épaule droite (HSH1-92.049). Le pan de la sanghātī qui recouvre le bras gauche jusqu'à l'avant-bras est visible dans la plupart des cas, il est terminé sur le genou gauche par trois arcs de cercles formant un feston. On retrouve ces détails vestimentaires sur les grands Buddha en bronze datant de la même période (XVIII ${ }^{\mathrm{C}}$ siècle) qui sont installés dans les monastères contemporains. L'antaravāsaka (vêtement inferieur) est indiqué par incision en bordure supérieure et inférieure, la bordure inférieure dégageant les chevilles et les pieds. Parfois, mais assez rarement, la ceinture est indiquée. Étant donné que chaque pièce a été travaillée individuellement, la physionomie des visages est toujours différente. Cependant, certaines caractéristiques demeurent semblables. Les yeux sont toujours soit grand ouverts, soit mi-clos. Les pupilles sont toujours indiquées par le percement à la pointe du revêtement métallique (signe de l'existence d'une cérémonie d'ouverture des yeux). Les sourcils sont exagérés et occupent une grande partie du front, représentés en arc de cercle ou en arc circonflexe, continu ou discontinu. Les nez sont petits, parfois droits ou à peine busqués, contrairement à ce que l'on peut observer sur des pièces en bronze de taille supérieure et de même période, dont le nez est, par contre, nettement busqué ${ }^{4}$. Les narines sont en règle générale assez épatées et elles sont toujours soulignées par une incision en virgule profondément marquée. La bouche, de taille très variable a, la plupart du temps, les commissures relevées. Les oreilles sont de trois types, pointues aux lobes longs, arrondies aux lobes longs ou pointues aux deux extrémités et recourbées. Le percement pour les boucles d'oreilles est figuré par une longue incision verticale qui ne transperce cependant pas le métal. Le traitement de la coiffure varie : il s'agit soit de boucles traitées en cercles incisés, soit d'un quadrillage formant ainsi des rangées de losanges. La hauteur et la largeur de la partie sommitale pointue sont également très variables. Il s'agit probablement de l'uṣnīṣa (protubérance crânienne ou Joyau), qui peut être soit conique, soit en forme de bulbe, surmonté d'une pointe. Dans la plupart des cas, il est laissé lisse, mais il peut parfois être couvert de quadrillages en losanges ou de festons pointus. Sa base peut être droite ou renflée, formant ainsi un ou plusieurs anneaux successifs. Là encore, la décoration varie, cercles incisés ou quadrillages en losanges, elle peut également être lisse.

Au cou, les plis de beauté sont en règle générale indiqués, soit simples, soit redoublés. $\mathrm{Au}$ niveau du torse, les pectoraux sont figurés en un volume légèrement pointu et les

4. Madeleine Giteau, « Note sur les pièces d'art bouddhique de la collection de S. M. le Roi du Laos ", Arts asiatiques, 30, 1972, p. 91-128. 
mamelons sont toujours indiqués par un cercle incisé. Une grande attention a été portée à la réalisation des mains. Le pouce est différencié des autres doigts, plus long, sa base est soulignée par une ou deux incisions en virgule, l'ongle est parfois noté par une incision en arc de cercle. Les phalanges des autres doigts sont toujours soulignées par une ou deux incisions transversales marquant les pliures des doigts. Les jambes sont croisées en vìrāsana (jambe droite sur jambe gauche). Les pieds ont été l'objet d'un même soin, le talon est indiqué par une virgule, le gros orteil est différencié, son ongle est parfois figuré de profil, et les phalanges des orteils sont délimitées par des incisions, simples ou doubles. Sur deux images seulement, la Roue de la Loi est incisée sur la plante du pied droit (seul visible), sous la forme d'un cercle simple (HSH1-92.013 et 014).

\section{Les socles}

À la différence des Buddha, dont la taille ne varie pratiquement jamais par rapport à la taille de leurs socles respectifs, on peut définir trois sortes de socles, deux formes hautes et une forme basse. Douze types de socles ont été définis, qui peuvent se répartir en trois groupes (voir fig. 12, 13 et 14). Les cinq premiers types (types 1 à 5) correspondent à des socles en forme de cloche, resserrés vers le haut et s'évasant vers le bas en ovale. Le second groupe est constitué de socles droits, qui s'évasent également vers le bas (types 6 à 8). Le troisième groupe (types 9 à 12) représente des socles bas, en forme de cloche, évasés à la partie inférieure. Pour chacun de ces trois groupes, le nombre de registres est variable (nous entendons par « registre " l'espace étagé des décors qui ornent ces socles). Pour les socles du groupe un, les registres varient de un à cinq, pour le groupe deux, de cinq à six, pour le groupe trois, de un à cinq. Le premier registre de tous ces socles est toujours isolé des autres par une moulure à arête médiane saillante, qui marque un resserrement avant l'évasement du socle. Nous avons défini les types en fonction de leur fréquence. Ainsi, le type un correspond à vingt-huit pièces, le type deux à vingt-trois, le type trois à dix-huit, le type quatre à dix, le type cinq et le type six à deux, le type sept à un, de même que les types huit, dix, et douze. Le type neuf concerne quatre images, et le type onze, trois Buddha.

\section{Les décors}

Les décors des socles sont extrêmement riches. Pour 108 images, nous avons défini soixante-six frises décorées, séparées par des bandeaux ornés, au nombre de dix (voir fig. 15 à 20). Ces frises et ces bandeaux se déroulent tout autour du socle. Dans certains cas, la face postérieure peut constituer une variante de la frise incisée sur la face antérieure, en règle générale par la simplification des fioritures qui accompagnent le motif principal (voir frises $11,40,62,63$ et 64 sur les figures sus indiquées). Comme on peut le remarquer sur ces schémas, qui reprennent une séquence du motif de chaque frise, cette séquence peut être plus ou moins longue et complexe (voir par exemple la frise 11, particulièrement ornementéc). Il n'est pas dans notre propos ici de décrire en détail chacune de ces frises, puisque les dessins en sont donnés, mais il nous semble pouvoir les réunir en trois groupes principaux.

La majorité des motifs, qui constituent le groupe un (frises 1, 2, 5 à 10, 13 à 16, 35, $36,41,47,49$ à 58,66 ), dérivent de la forme du pétale de lotus. Ce motif floral est plus ou moins stylisé ou géométrique et les détails ornementaux qui l'accompagnent sont plus ou moins nombreux et complexes. Il peut se terminer en pointe ou être de forme arrondie et être plus ou moins fermé ou ouvert. Dans plusieurs cas, les étamines du lotus sont indiquées entre les pétales par des stries parallèles verticales. Dans un cas (frise 66), les pétales sont reliés entre eux par un quadrillage purement géométrique. Dans la grande 
majorité des cas (sauf pour la frise 36), le bord extérieur du pétale est constitué par un liseré lisse. La partie interne du pétale peut être laissée vierge ou être ornée de la forme générale du pétale reprise en plus petit, et la bordure ornée de bandes de points, de stries, voire encore de virgules.

Les frises du second groupe, qui sont d'ailleurs les plus complexes (frises $3,4,11,12$, 37 à 40,62 à 64), plutôt que d'être inspirées directement de la fleur de lotus, figurent plus probablement des motifs végétaux stylisés, déroulant des crosses de feuillages entre lesquelles peuvent apparaître des fleurs simples, pour la plupart quadrilobées. Mais les crosses de feuillages peuvent être dans certains cas particulièrement stylisées et n'être plus qu'un simple trait terminant par des boucles (frises 3 et 4 ).

Le groupe trois concerne les motifs purement géométriques (frises 18, 42, 44 à 46, 65). La frise 18 constitue un type à part, qui reprend une succession d'éléments quadrilobés étirés, ornés au centre d'une courte spirale, séparés tous les trois éléments par des motifs en virgule. Les autres frises sont constituées par des suites de triangles ou de losanges plus ou moins décorés en bordure et dans l'espace interne de stries, de cercles ou de virgules.

Les bandeaux qui viennent s'interposer entre les frises sont beaucoup plus sobres (voir figure 19). Ils sont tous géométriques (ils ont été numérotés de $\mathrm{A}$ à $\mathrm{J}$ ). Il s'agit la plupart du temps de bandeaux de perlages incisés encadrés par des filets simples, doubles ou triples. Les bandeaux de perlages peuvent être remplacés par des stries verticales, des dents de scies ou des chevrons, verticaux ou horizontaux.

\section{Fréquence des décors sur les types de socles}

Après avoir isolé tous ces décors sur chacun des Buddha, il importait de définir si des séries se dessinaient en fonction des différents types de socles. Étant donné que de nombreux Buddha ne sont conservés que très partiellement, et que certains types sont représentés par un nombre trop faible de pièces et parfois par une unique image, cette tentative de sériation n'est donnée qu'à titre indicatif. Mais elle sera utile lorsque nous pourrons, dans un avenir que nous espérons proche, comparer ces pièces à celles qui sont conservées à Luang Prabang et dans certaines collections particulières, à Vientiane. Le grand nombre de motifs que nous avons isolé permet, par rapport au nombre de registres présent sur chaque pièce, une multitude de possibilités et de combinaisons des motifs entre eux. La plupart du temps, les deux premiers registres, séparés par la moulure à arête médiane saillante, figurent deux frises inspirées de la fleur de lotus, inversées l'une par rapport à l'autre. Sur les registres inférieurs, les motifs peuvent être très différents. Le dernier registre est souvent laissé vierge mais, dans dix-huit cas, une inscription y prend place. Nous reviendrons plus loin sur ce sujet. Cependant, pour quelques types, qui sont par ailleurs les plus fréquents, certains décors reviennent de manière semblable ou très proche, avec des variantes qui apparaissent essentiellement au niveau des bandeaux.

Pour les vingt-cinq Buddha de type 1, quatre décors reviennent, identiques. Sur six Buddha $\left(\mathrm{n}^{\circ} 9,68,72,138,185\right.$ et 186$)$, on retrouve le décor suivant : deux frises de type 2 suivies d'un bandeau de type $B$, suivi d'un registre vide, d'un bandeau de type I, d'une frise de type 8 , terminée par un bandeau de type A. Sur six autres $\left(n^{\circ} 57,63,65,67,132\right.$ et 137), le décor est représenté par deux frises de type 1, suivies d'un bandeau de type $B$ et de type I séparées par un registre vide, suivi par une frise de type 4, terminée par un bandeau de type A. Les six Buddha suivants $\left(\mathrm{n}^{\circ} 10,80,122,127,133,147\right)$ sont semblables aux précédents quant à la répartition du décor, sauf pour l'avant-dernière frise qui est du type 8 au lieu d'être du type 4 . Les deux derniers Buddha concernés par un même décor ( $n^{\circ} 26$ et 47 ) ont deux frises de type 1, suivies d'un bandeau de type B puis de 
type I séparées par un registre vide, terminé par une frise de type 3. Dans ce cas précis, ce que l'on ne percevait pas dans les séries précédentes, les détails iconographiques du Buddha sont semblables également (mêmes incisions en bordure du vêtement, au cou, aux mains et aux pieds, le joyau est très haut avec une base ornée de quadrillages). Ils sont cependant de taille différente, comme l'était celle des trois séries de six. Les autres images de ce type ont toutes des décors très différents les uns des autres.

Vingt-deux Buddha sont du type deux. Quatre $\left(\mathrm{n}^{\circ} 32,52,59,66\right)$ présentent le décor suivant : deux frises de type 2 suivies par deux bandeaux de type B séparés par un registre vide. Leur taille et les caractéristiques iconographiques des Buddha varient. Sur deux autres ( $\mathrm{n}^{\circ} 55$ et 136 ), on trouve deux frises de type 32 , suivies d'un bandeau de type B, puis à nouveau d'une frise de type 32 terminée par un bandeau de type A. Dans ces deux cas, les Buddha ne sont pas conservés.

Pour les Buddha de type 4 , au nombre de dix, une série revient à six reprises $\left(n^{\circ} 11\right.$, $42,98,101,125,135$ ). Le décor est plus simple, un registre libre suivi d'une frise de type 7 , terminée par un bandeau de type B. Les détails iconographiques varient, mais ils sont tous de taille moyenne.

Enfin, pour le type 11 , qui concerne quatre Buddha, deux ( ${ }^{\circ} 4$ et 71 ) présentent un décor formé à base de frises et de bandeaux de même type bien qu'ils ne soient pas rigoureusement semblables. Le premier possède deux bandeaux de type A suivis d'un registre libre, de deux bandeaux de type A encadrant une frise de type 50, se terminant par deux bandeaux de type $\mathrm{A}$ encadrant un registre à nouveau vide. Le second présente deux bandeaux de type A qui encadrent deux registres vierges successifs, suivis d'une frise de type 50 terminée par un bandeau de type A. Ces deux Buddha sont de petite taille et, bien que leurs caractéristiques iconographiques ne soient pas toutes semblables, certaines présentent des points communs : l'indication de la marque des plis sur l'antaravāsaka, la même bordure inférieure de l'uttarāsanga, la bouche, petite mais aux commissures largement relevées. La coiffure est traitée en losanges et le Joyau, conique.

Les Buddha des autres types possèdent tous des décors très différents les uns des autres. Mais il est possible que des Buddha conservés dans d'autres collections présentent des caractéristiques communes avec ceux-ci. À terme, cette étude des décors, qui peut paraître de prime abord rébarbative, pourrait permettre de mieux connaître certains ateliers de fabrication, voire de repérer la main de certains artistes, qui auraient pu avoir l'habitude de reproduire plus particulièrement certains décors et détails iconographiques.

\section{Les techniques de fabrication}

Toutes ces pièces ont un revêtement en or ou en argent. Le revêtement peut être totalement en or ou totalement en argent, mais il peut aussi être mixte, le revêtement du Buddha étant dans ce dernier cas en or et le revêtement du socle en argent.

\section{Le moulage des plaques}

La première opération semble être le moulage des plaques d'or ou d'argent qui constituent le revêtement extérieur des Buddha. Des demi-moules, probablement en bois, ont été utilisés pour emboutir les plaques antérieures et postérieures qui forment, une fois réunies, la forme générale du Buddha. Le corps du Buddha, à l'exclusion de la flamme ou du bulbe, devait être moulé en même temps que le socle, qu'il s'agisse d'une plaque unique ou de deux plaques (lorsque le métal est mixte, or et argent). Dans ce cas, les plaques se juxtaposent de quelques millimètres l'une sur l'autre dans le sens horizontal. Une fois embouties, ces demi-plaques sont réunies par un système de languettes formant 
sur les côtés une série de chevrons verticaux (voir fig. 3). Par la suite, sur des Buddha plus récents mais de même type, la fermeture des plaques en chevrons sera abandonnée au profit d'une fermeture droite, les plaques se chevauchant légèrement dans ce cas-là dans le sens vertical. Certains éléments, tels que les oreilles et la partie sommitale, sont rajoutés par la suite, probablement après avoir été décorés (voir fig. 5). La flamme consiste en une plaque en forme de triangle, qui, une fois enroulée sur elle-même et décorée, forme un cône qui vient s'emboîter dans la base du Joyau quelle que soit sa forme, droite ou en bulbe. Les oreilles consistent en une plaque prédécoupée selon la forme que l'on veut donner à l'oreille, puis décorée à plat avant d'être mise en place par un système de languette rectangulaire, qui passe dans une fente ménagée dans les grandes plaques, sur les côtés de la tête (voir fig. 5). Enfin, sur les Buddha en argent, et seulement sur ceux-ci, la base du socle est renforcée parfois par un fil d'argent, soudé au socle et qui l'entoure complètement (voir fig. 6).

Avant d'avoir été placés dans la cache du dépôt de fondation, certains Buddha ont dû avoir une existence à l'air libre relativement longue puisque certains ont été abîmés, puis ont fait l'objet d'une restauration. L'un d'entre eux, par exemple, a subi une restauration entre le bras gauche et le buste : une petite plaque d'or a été installée à cet endroit-là pour combler un manque (voir fig. 5 , où le raccord se voit nettement).

\section{Le keson dokmai}

Lorsque les deux plaques qui constituent le Buddha sont réunies (peut-être sans les éléments rajoutés, ou tout du moins sans les oreilles), cette forme vide est placée à l'envers, base du socle vers le haut, de manière à recevoir le keson dokmai. D'après l'aspect des strates que l'on peut observer sur les Buddha qui ont perdu leur revêtement en métal, le keson dokmai devait être à l'état liquide lorsqu'il a été coulé dans ces formes. Parfois, la pâte présente des circonvolutions remplies de bulles d'air, qui laissent à penser qu'elle n'était pas assez fluide lorsqu'elle a été coulée (voir fig. 7). L'âme de certains Buddha a été coulée en deux temps, d'une part le corps du Buddha, et d'autre part le socle. Dans ce cas-là, lorsque le revêtement de métal s'est détérioré, les deux parties se sont détachées parfaitement, sans présenter de cassure (voir fig. 8). Des impressions de tissu sont visibles dans le keson dokmai encore mou, sur la face inférieure du socle (voir fig. 6). Plusieurs hypothèses sont possibles. Elles ont pu être faites par l'application d'un linge sur le keson dokmai encore liquide dans la forme encore en position à l'envers, mais cette marque a pu s'imprimer lorsque le Buddha a été remis en position à l'endroit, posé sur un linge qui a pu protéger la base lors du décor extérieur. Ces marques de tissu témoignent d'étoffes assez brutes et épaisses, à la trame assez lâche telle que pourrait en laisser une toile de jute (voir fig. 6). Le fil de chaîne et le fil de trame sont assez épais et de diamètres apparemment semblables. Pour consolider la solidité de la pièce, un bâtonnet de bambou est inséré au centre de la forme. Le bambou a disparu dans tous les cas mais ses traces sont nettement visibles dans le keson dokmai (voir fig. 9). Pointu à son extrémité supérieure, il s'enfonce dans l'âme du Buddha jusqu'à la pointe, ce qui laisse à penser que la partie sommitale, même en tant qu'élément rapporté en métal, était en place dans le moule avant l'emboutissage du métal, puisqu'elle est, elle aussi, comblée par du keson dokmai.

La composition de cette pâte peut être enrichie de paillettes d'or, dont certaines sont visibles en surface sur certaines pièces. Cependant, nous ne connaissons pas le mélange exact des matériaux utilisés pour fabriquer le keson dokmai à cette époque. Cette technique est encore connue et a été employée jusqu'à il y a une quarantaine d'années. Dans les villages d'artisans de ce type que nous avons pu visiter dans la région de Champassak, qui sont également des bronziers, certains vieux artisans se souviennent 
avoir utilisé cette technique et ont pu nous en fournir la composition actuelle. Il s'agit d'un mélange de résine végétale (nan man niang) provenant d'un arbre appelé tabeng, d'argile et de pétrole, et, pour l'essentiel, de fleurs sauvages très odorantes, blanches ou jaunes (dok khaniom - ces fleurs n'ont pas été identifiées - et dok khun - canéficiers), provenant d'arbres poussant dans la forêt, séchées puis pilées pour être transformées en poudre. Ces fleurs sont choisies à l'exclusion de toute autre parce qu'elles sont considérées comme des fleurs sacrées, réputées pour leur fragrance, mais aussi parce qu'elles sont très riches en pollen. Cependant, les analyses polliniques pratiquées sur des échantillons ramenés en France provenant de Buddha en keson dokmai de Huay Sa Hua 1 n'en ont malheureusement révélé aucune trace. La résine végétale, qui sert également de base aux peintures utilisées pour rchausser les monastères, fait ici office de liant. Les techniques d'utilisation actuelles semblent différer des techniques anciennes. Le keson dokmai est chauffé de manière à pouvoir être malaxé, sans être à l'état liquide. La forme, limitée aux traits essentiels de l'aspect du Buddha et du socle, assez fruste, est faite à la main ou bien elle est placée dans un moule en bois en deux parties. Elle n'est plus recouverte d'un revêtement métallique, mais enduite de peinture dorée ou recouverte de feuilles d'or, sans détails ni décor particulier, bien que les revêtements métalliques aient été encore connus au début du vingtième siècle et façonnés par les artisans bijoutiers du village de Vat Luang $\mathrm{Kau}$, situé à quelques kilomètres du site de Vat Phu. Malheureusement, aucun artisan encore vivant n'a pu nous fournir de détail sur les techniques utilisées et les moules en bois ont tous disparus. Lorsque l'on compare la qualité du keson dokmai actuel avec celle de l'ancien, la pâte est beaucoup plus friable et sableuse. Il n'existe de ce fait pratiquement plus de Buddha fabriqués dans cette matière à l'heure actuelle, à cause de leur fragilité, ce qui leur confère quelques dizaines d'années d'existence au plus. De ce fait, cette technique a été abandonnée. Nous n'avons pu en voir que deux, conservés dans le trésor de monastères de la région, à Champassak et à Vat Muang Kau. Le premier, seulement doré à la feuille (petites feuilles extrêmement minces, carrées, dont on recouvre les statues des Buddha lors des cérémonies), conserve des traces de moule sur les côtés et le bâton de bambou est visible au niveau de la flamme, brisée. Le second, façonné à la main également autour d'un bâtonnet de bambou, ne possède, comme seule indication iconographique, que le pan du manteau, incisé sur le devant et dans le dos, tandis que le visage est à peine modelé.

\section{Le décor externe}

Sur tous les Buddha retrouvés à l'intérieur du dépôt de fondation, c'est la technique du défoncé qui a été utilisée pour le décor final. Cette technique consiste à graver le décor à la pointe à partir de la surface externe du métal. Les traits du Buddha, les détails anatomiques et vestimentaires, l'ornementation du socle, mais également les inscriptions qui ornent la base de certains, sont exécutés de cette façon. Le décor s'est incrusté dans le keson dokmai et s'est donc conservé, même si le revêtement en métal a disparu (voir fig. 1 et 2), ce qui suggère que le keson dokmai n'était pas complètement sec lors de l'étape finale. Rappelons, comme nous l'avons dit plus haut, que les yeux ont été percés, la pointe traversant la plaque de métal, ceci correspondant sans doute à une cérémonie d'ouverture des yeux des Buddha. 


\section{Les inscriptions}

Sur la totalité des Buddha de ce type retrouvés dans la cache sacrée, dix-huit pièces présentent une ou quelques lignes d'inscriptions. Elles ne dépassent pas les trois lignes. Certaines sont inscrites sur la partie antérieure du socle, d'autres en font le tour (voir fig. 10). Écrites en caractères tham, leur traduction définitive reste à faire et sera publiée dans la monographie exhaustive de cette fouille. Elles sont situées sur les registres non ornés des socles et, dans trois cas, sur la base inférieure du socle. Dans ce cas, il ne s'agit que de quelques caractères (voir fig. 11). Pour les plus courtes, il s'agit de noms propres, celui du donateur et parfois de l'artisan lorsqu'elles sont inscrites sous le socle. D'autres indiquent l'objet du don et/ou le nom de la personne pour qui et par qui a été fait le don et, parfois, en quelle occasion. La date exacte du don est dans plusieurs cas indiquée en termes lunaires (le jour et le mois). Seul un Buddha possède une date indiquant l'année : il est daté de 1668 (toutes les indications concernant ces inscriptions proviennent de communications personnelles fournies par Viengkèo Souksavatdy, qui s'est chargé de leur étude ; certaines sont à demi effacées et difficiles à lire).

\section{Conclusions provisoires}

De nombreux Buddha de ce type sont connus dans les collections conservées dans les monastères ainsi qu'au musée de Luang Prabang, d'autres dans des collections particulières à Vientiane, d'autres encore dans le centre du pays, que nous n'avons pas pu voir. À la même époque, des Buddha de type similaire ont circulé au Cambodge et en Thaïlande. Cependant, leur décor diffère. Cette collection de Buddha laotiens, située dans un contexte clos, permet de mieux cerner cet art. Ces pièces de petites dimensions sont considérées à l'heure actuelle comme provenant d'ateliers situés dans la partie nord du Laos, dans la région de Luang Prabang. Leur petite taille implique que ces pièces ont pu voyager sur tout le territoire du Laos ancien. Mais aucun site de fabrication n'est connu et il n'est pas impossible que des ateliers aient pu s'implanter ailleurs, dans le centre ou le sud du pays, tout en conservant le même style de décors. Cette étude reste à faire. Cependant, la richesse de ce dépôt de fondation témoigne de la renommée de ce monastère bouddhique. Ces pièces vécurent probablement de nombreuses années dans le trésor du monastère avant d'être enfouies dans ce dépôt sacré. Le fait qu'elles voisinent avec des bronzes de taille plus importante laisse à penser qu'il s'agissait peut-être de dons effectués plutôt par une classe sociale moyenne que par une classe sociale beaucoup plus aisée, leur technique de fabrication étant beaucoup plus facile et donc peut-être moins onéreuse que celle des Buddha en bronze; mais il s'agit là d'hypothèses qu'il reste à vérifier. Nous savons en tout cas que cette technique a perduré jusqu'au début du vingtième siècle. Une vingtaine de Buddha du même type ont été découverts par l'équipe du conservateur des monuments de Vat Phu dans la même région, enfouis en bordure du Mékong, dans une jarre en terre cuite. Tous en argent, leur facture et leur décor est beaucoup plus fruste et aucun ne possède d'inscription. La jarre qui les contenait, sans décor et à fond arrondi, d'un modèle courant utilisé jusqu'à nos jours, ne permet pas de donner de réelle indication de datation. Leur bon état de conservation pourrait permettre de penser qu'ils ne sont pas antérieurs à la fin du dix-neuvième siècle. Ces deux collections, qui ne sont pas encore exposées, sont conservées à Champassak et à Paksé en attendant de pouvoir être montrées. 


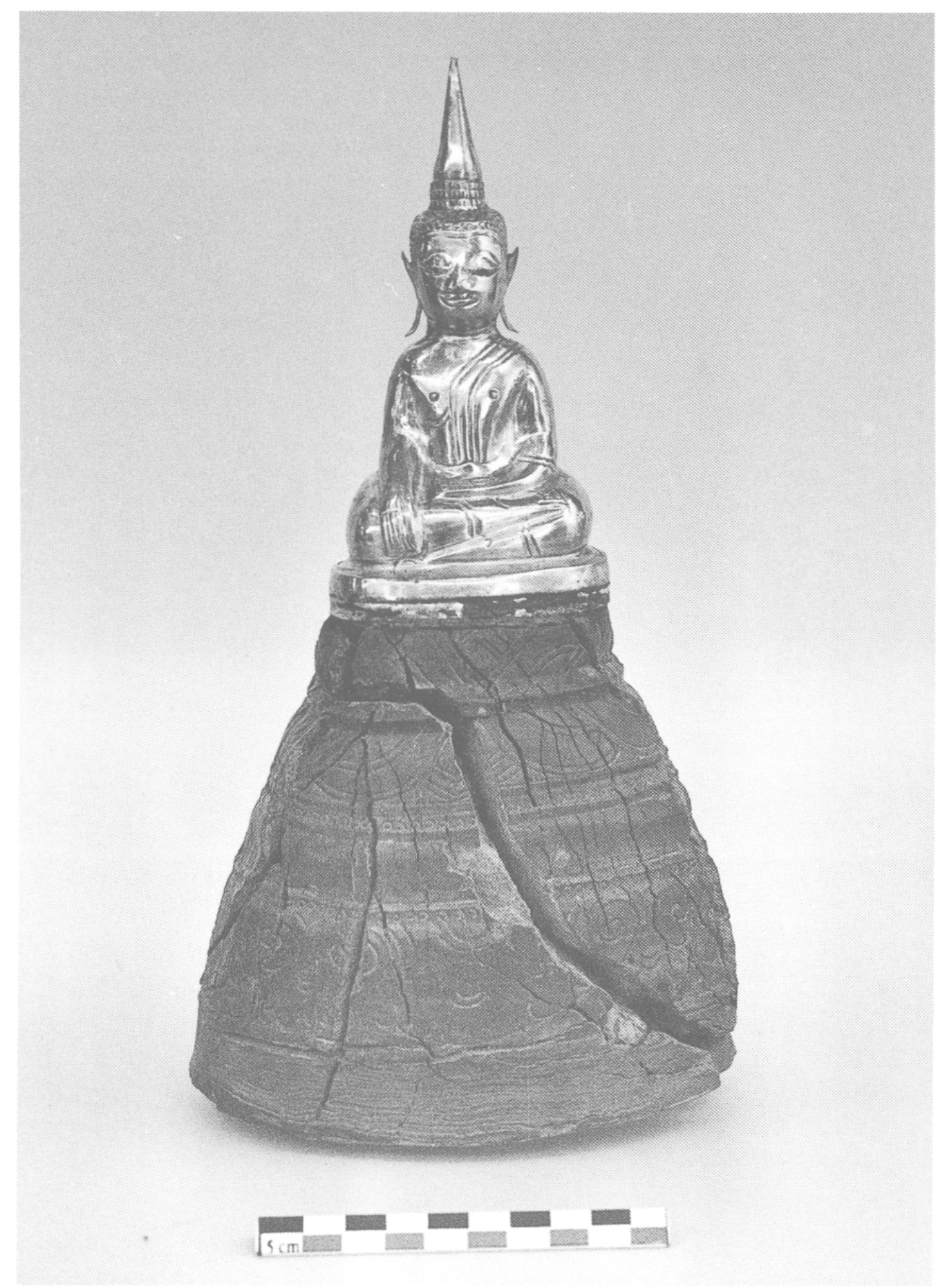

Fig. 1 - Buddha HSH1-92.008, vu de face.

(Photos : Marielle Santoni ; dessins : Jean-Pierre Message) 

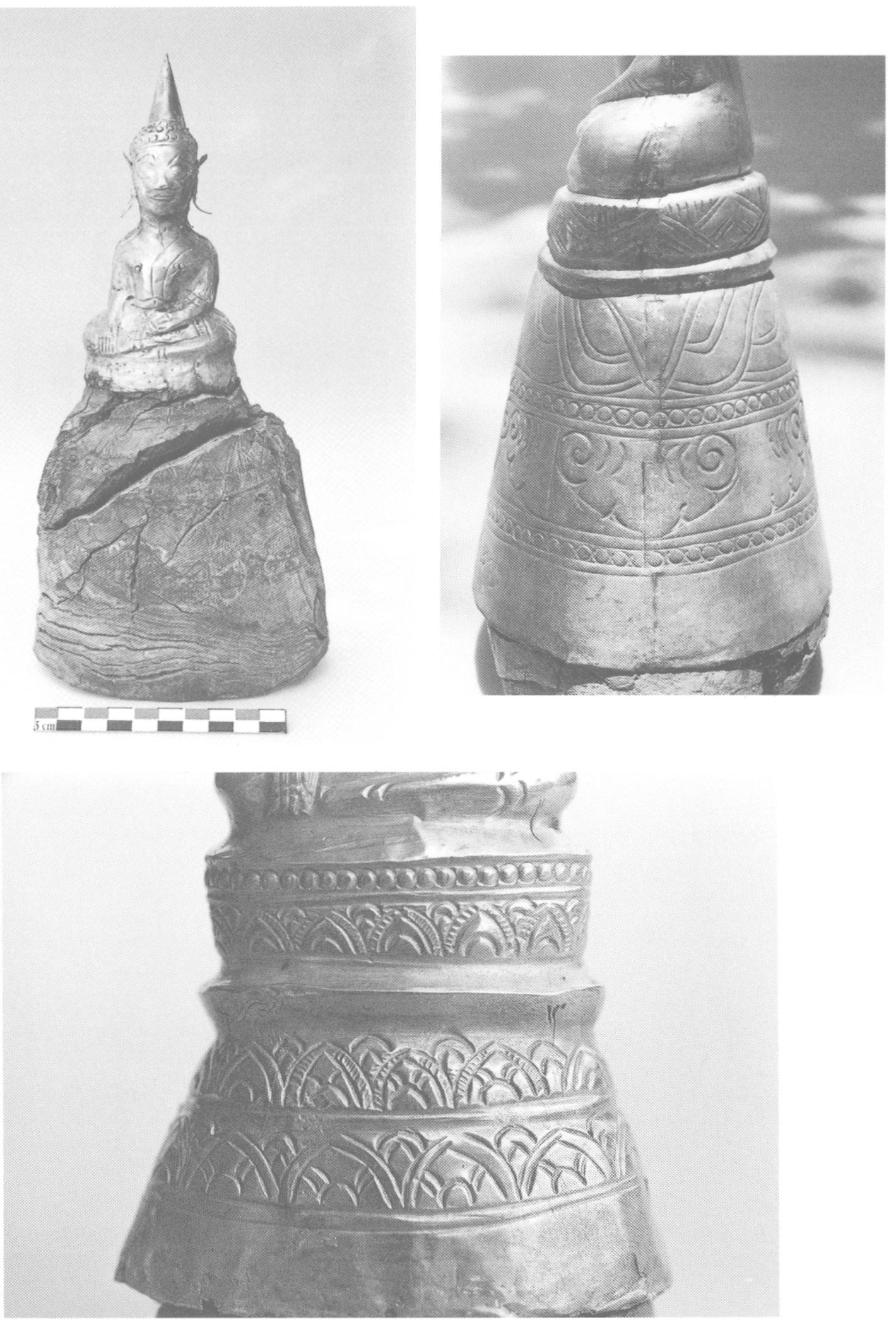

Fig. 2 - Buddha HSH1-92.046, vu de face.

Fig. 3 - Buddha HSH1-92.032, détail du socle, vu de face.

Fig. 4 - Buddha HSH1-92.048, détail de la fermeture des plaques. 


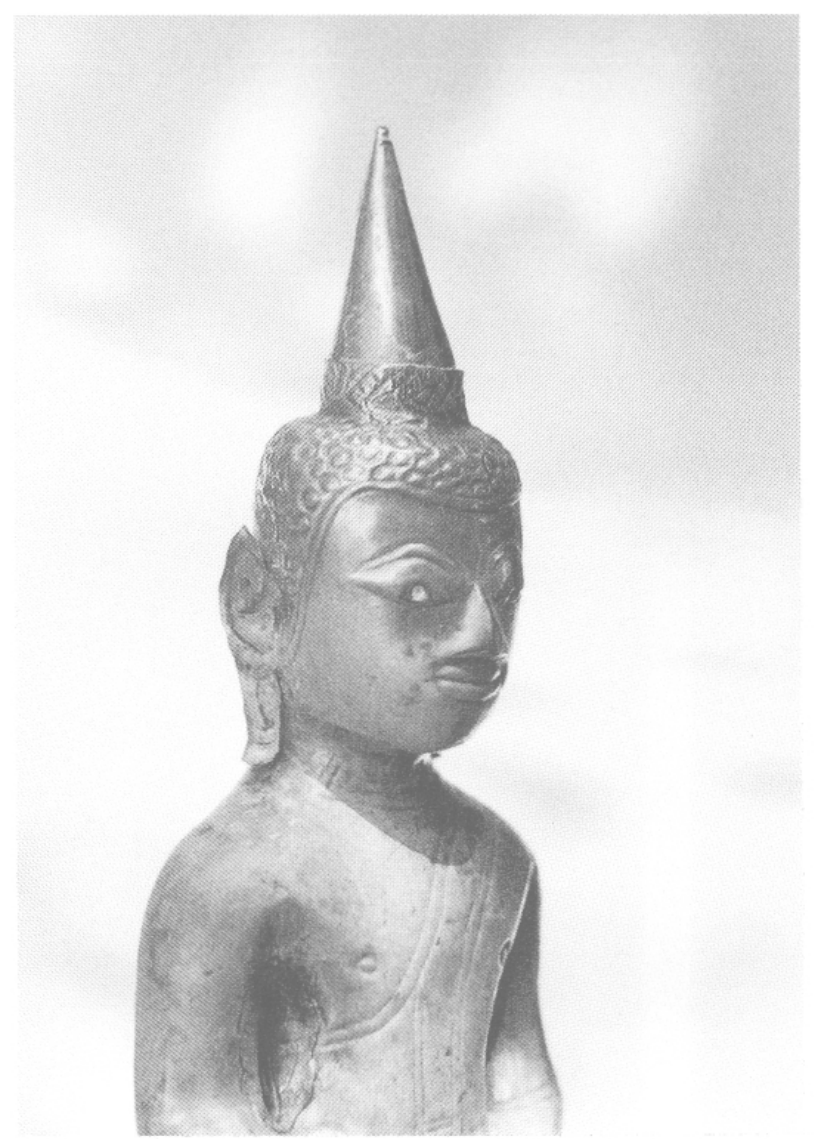

Fig. 5 Buddha HSH1-92.048, détail d'une restauration ancienne.

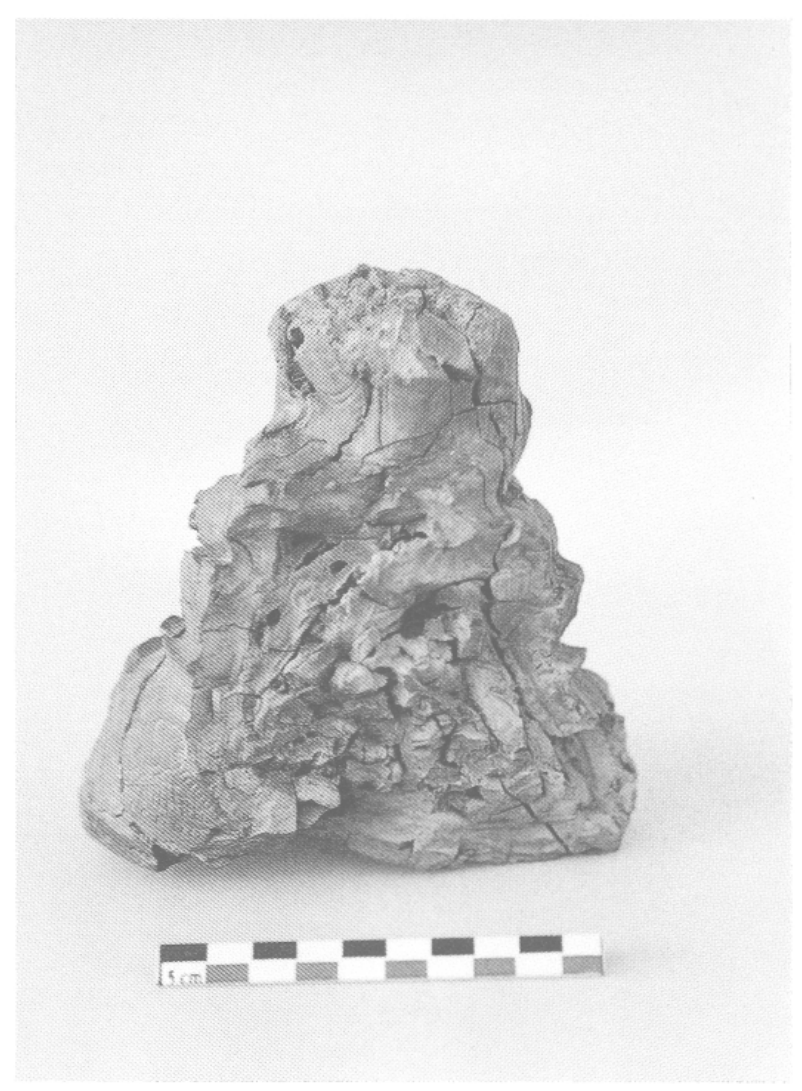

Fig. 7 -- Buddha HSH 1-92.128, détail du keson dokmai.

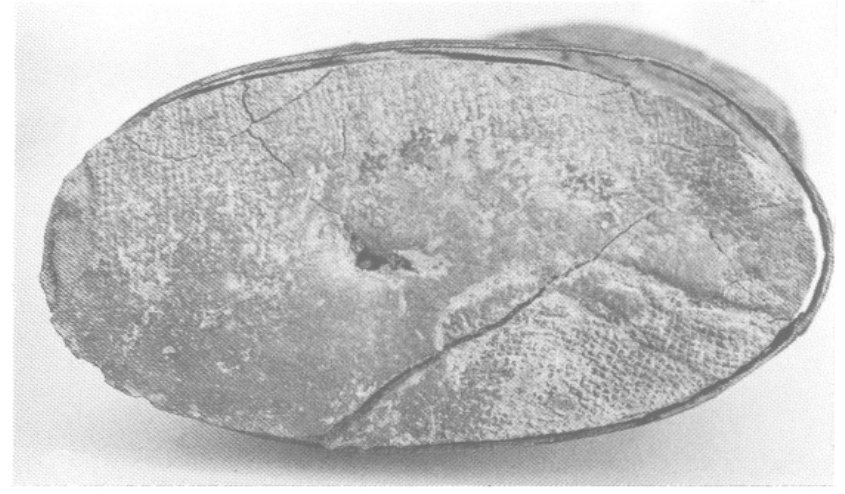

Fig. 6-Buddha HSH1-92.009, détail d'une impression de tissu sous le socle.

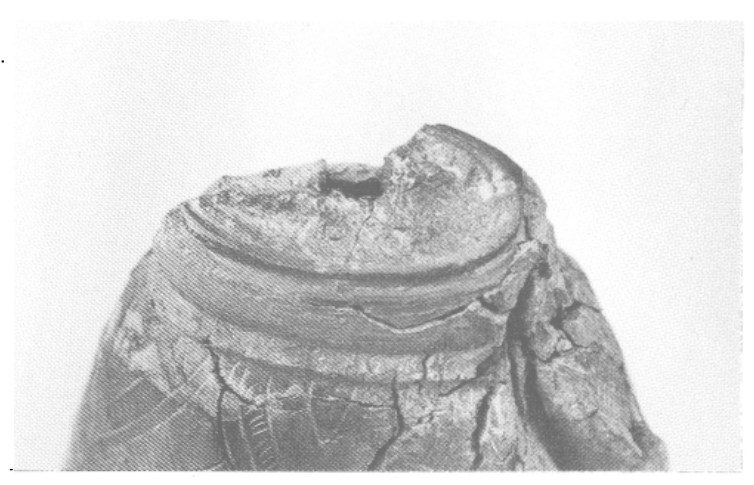

Fig. 8 - Buddha HSH1-92.136, détail d'une dissociation Buddha/socle. 


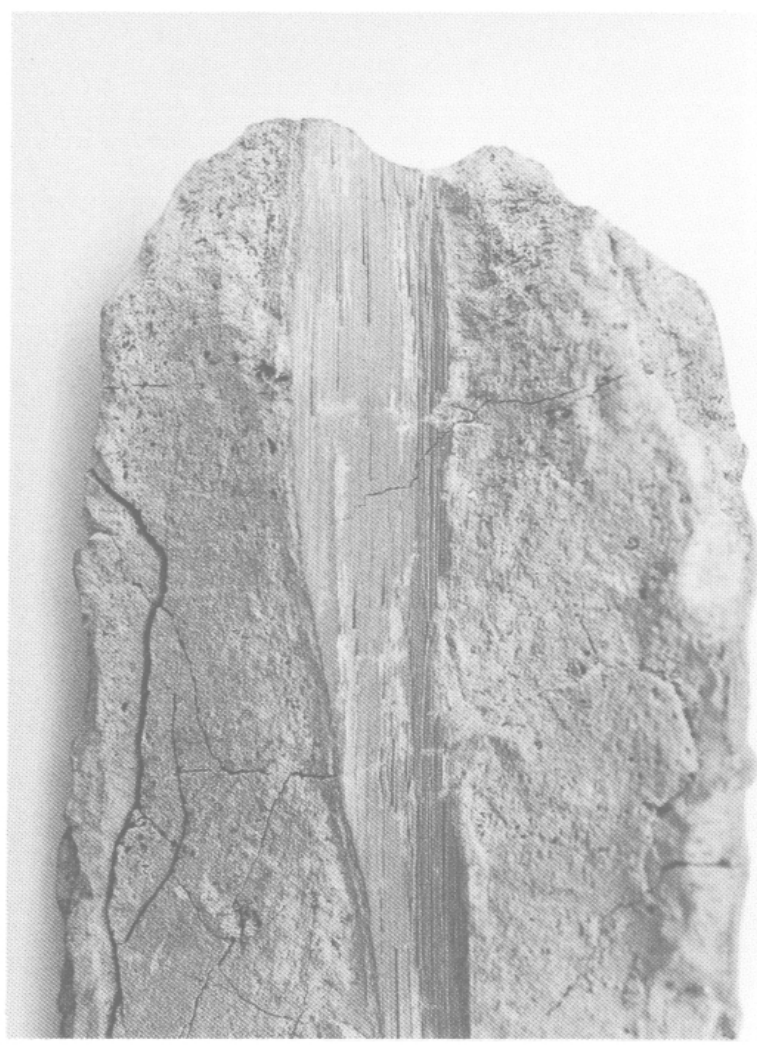

Fig. 9 Buddha HSH1-92.188,

détail de la trace du bambou interne.

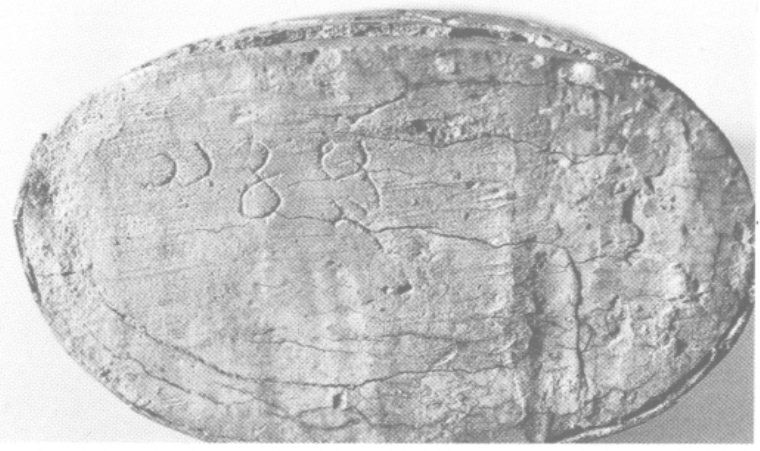

Fig. 11 - Buddha HSH1-92.092, détail d'une inscription sous le socle.

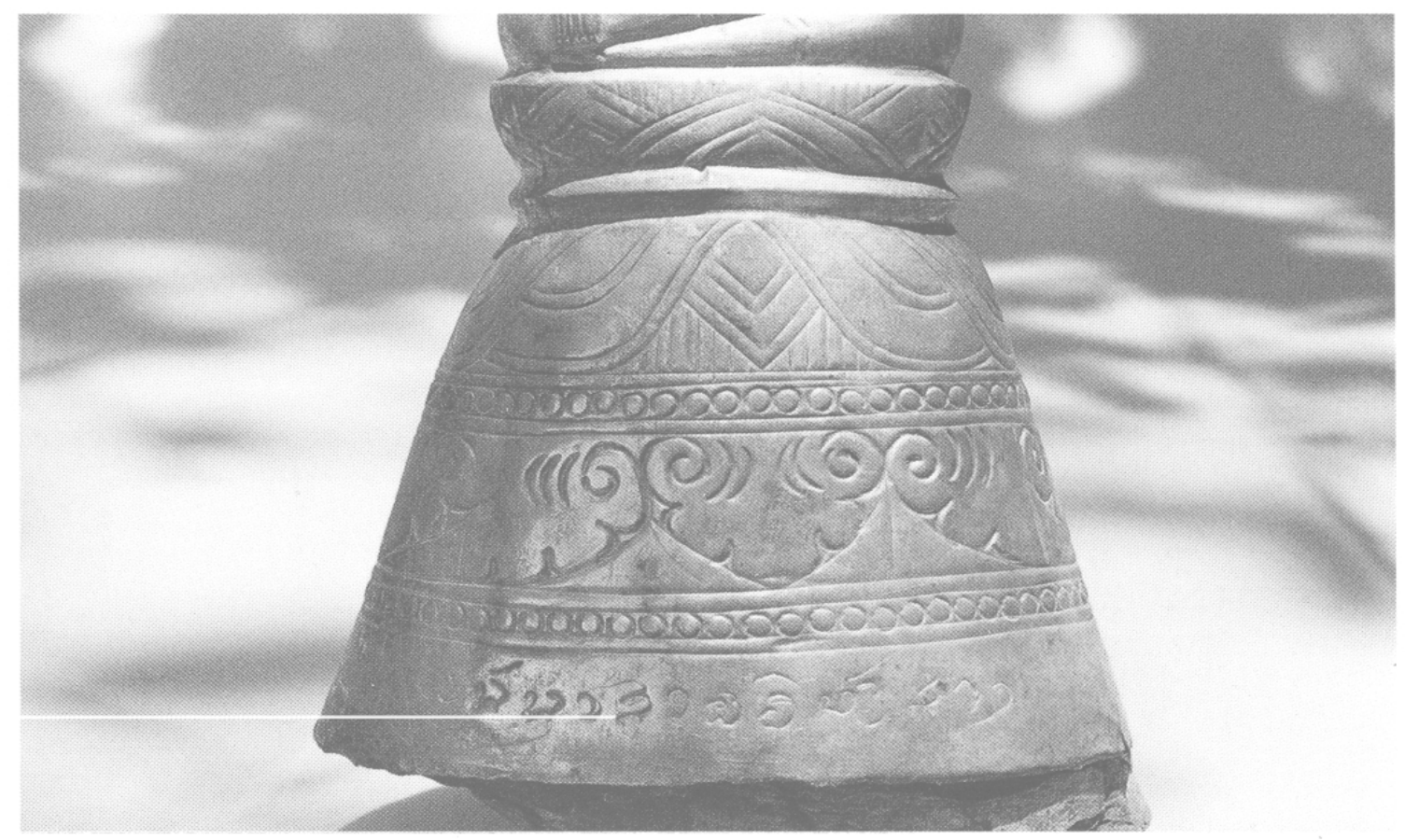

Fig. 10 Buddha HSHI-92.048, détail d'une inscription sur le socle. 


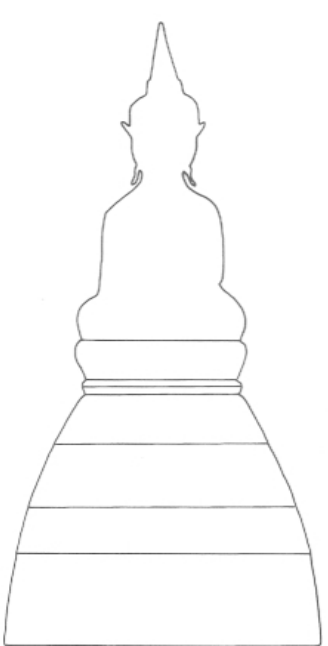

Trpe 1

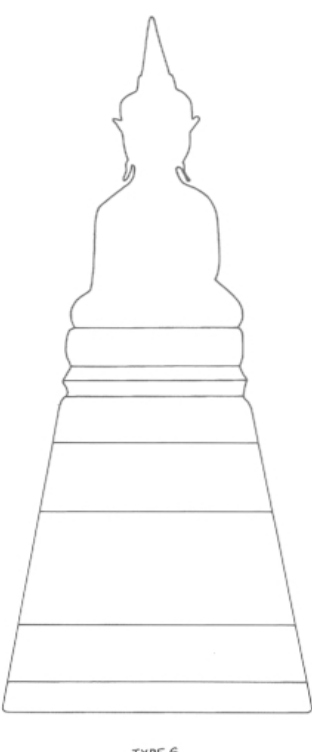

TYPE 6

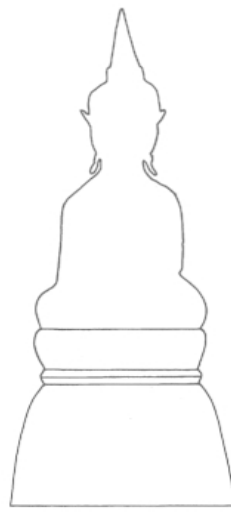

TYPE 12

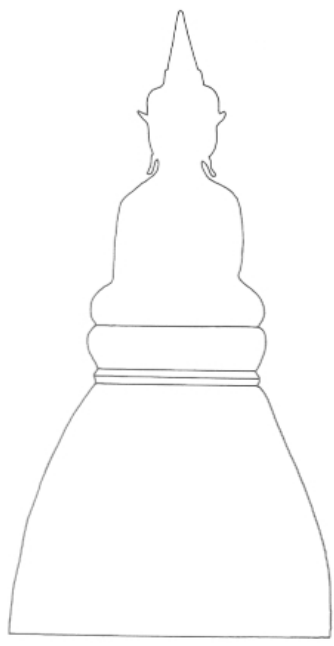

ryps

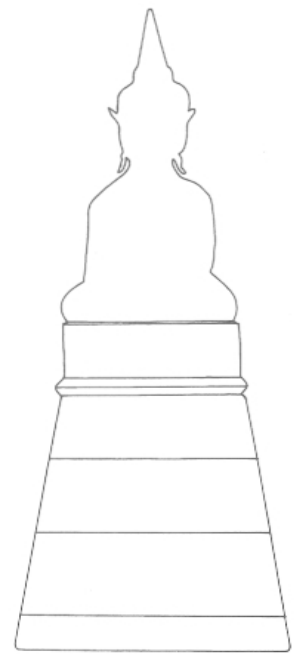

TYPE 7

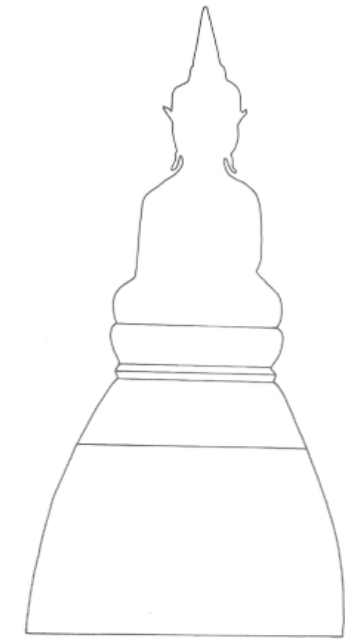

TPPE 3

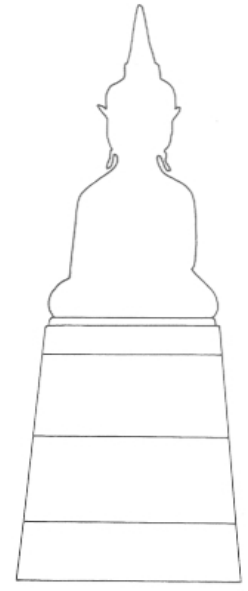

TYPE B

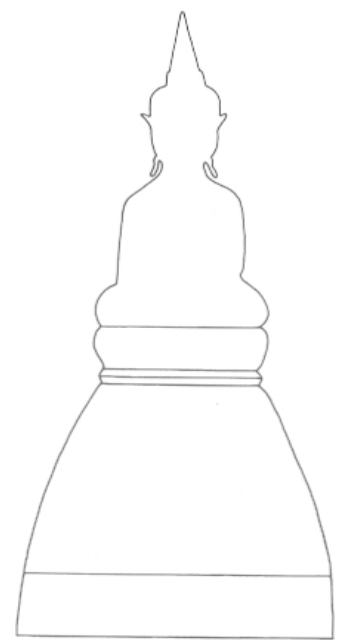

Typs 4

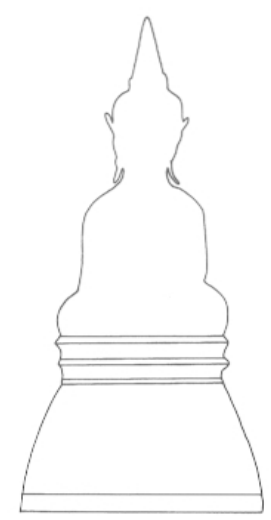

TYPE 11

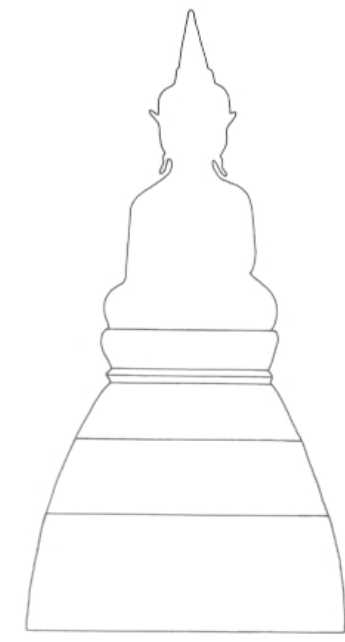

TYPE 2

Fig. 12, 13, 14 - Types de socles inventoriés 


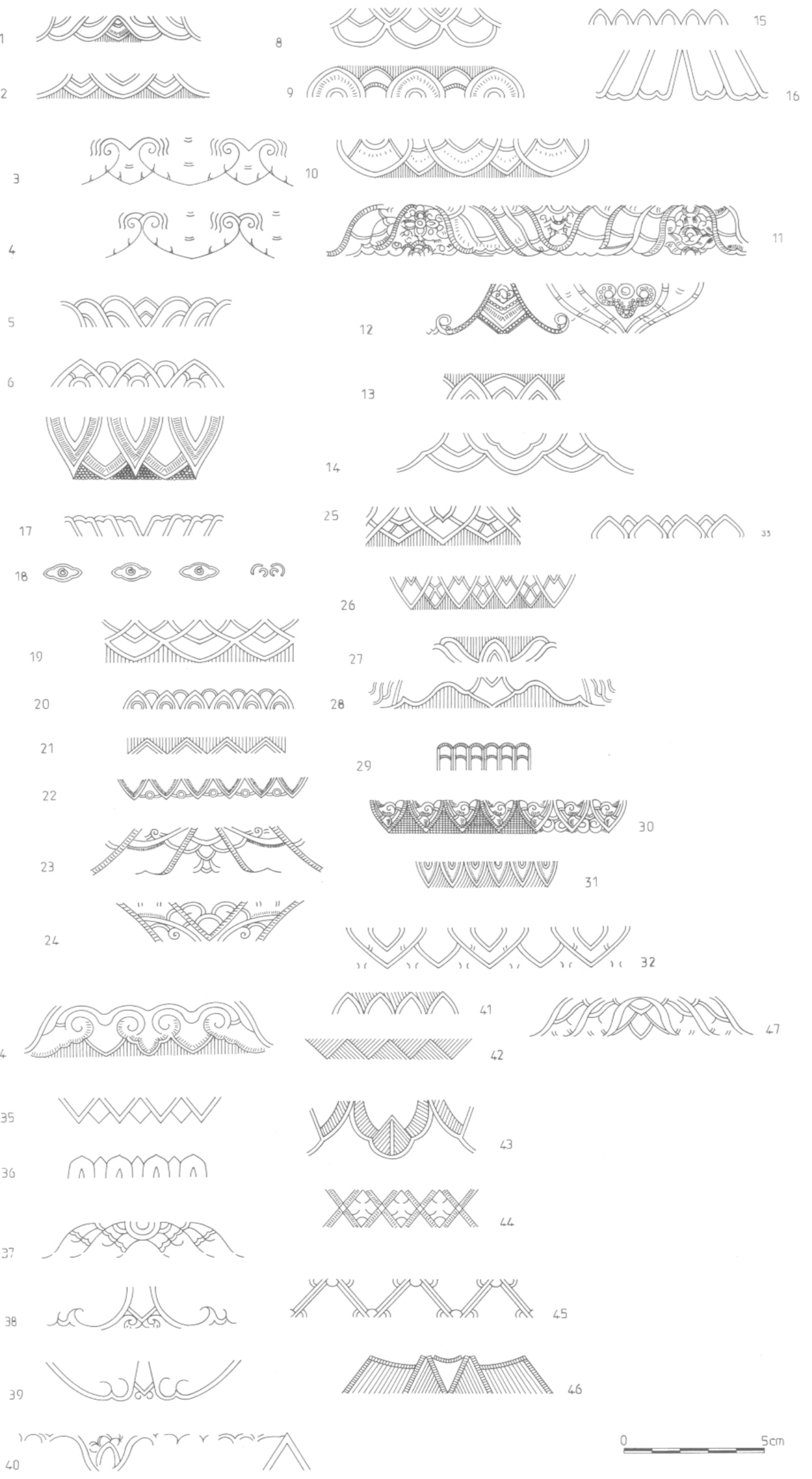




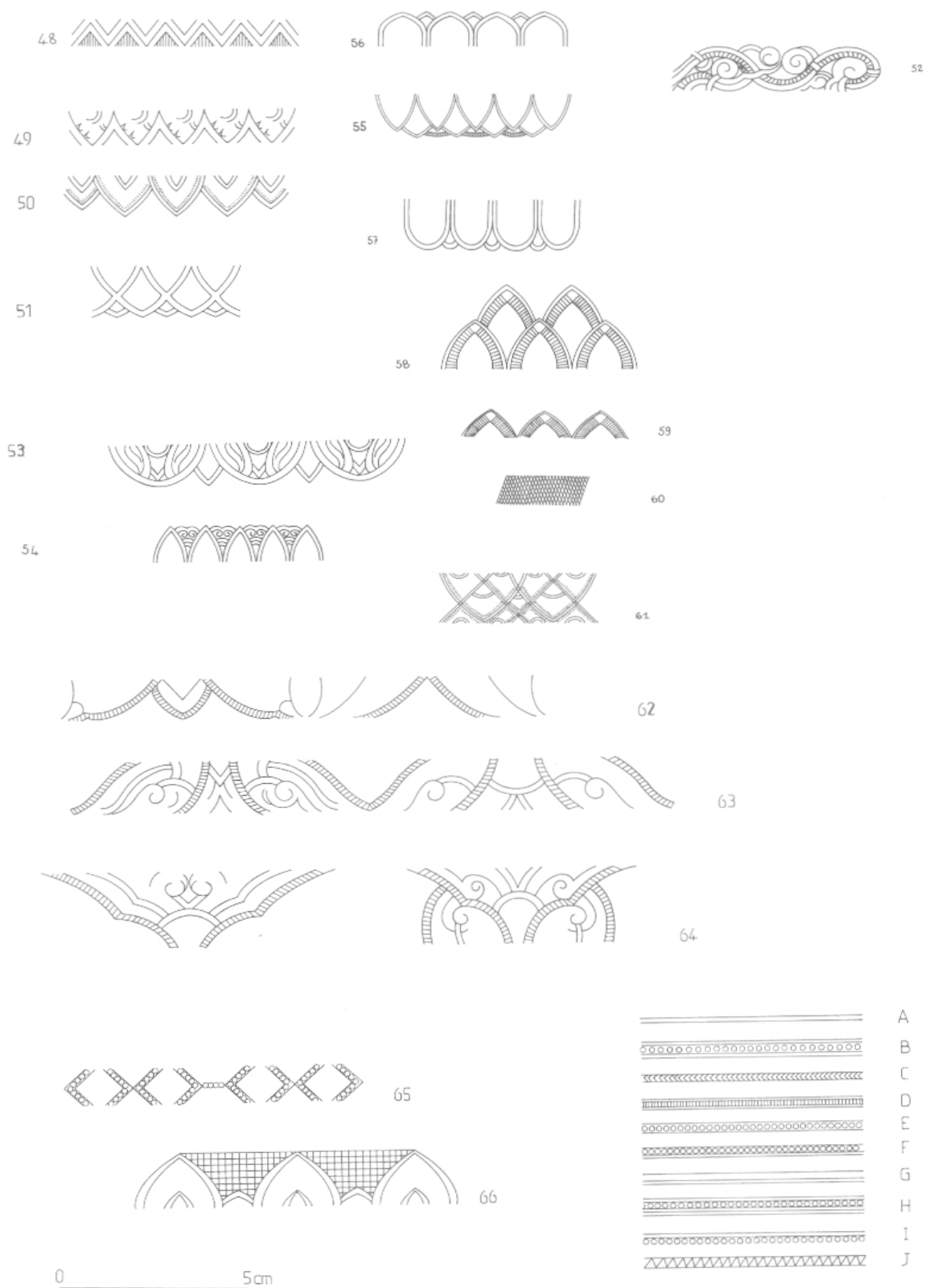

Fig. 15, 16, 17 (page précédente) et 18,19-Types de décors 